\title{
Test-retest reliability of two-dimensional video analysis during running
}

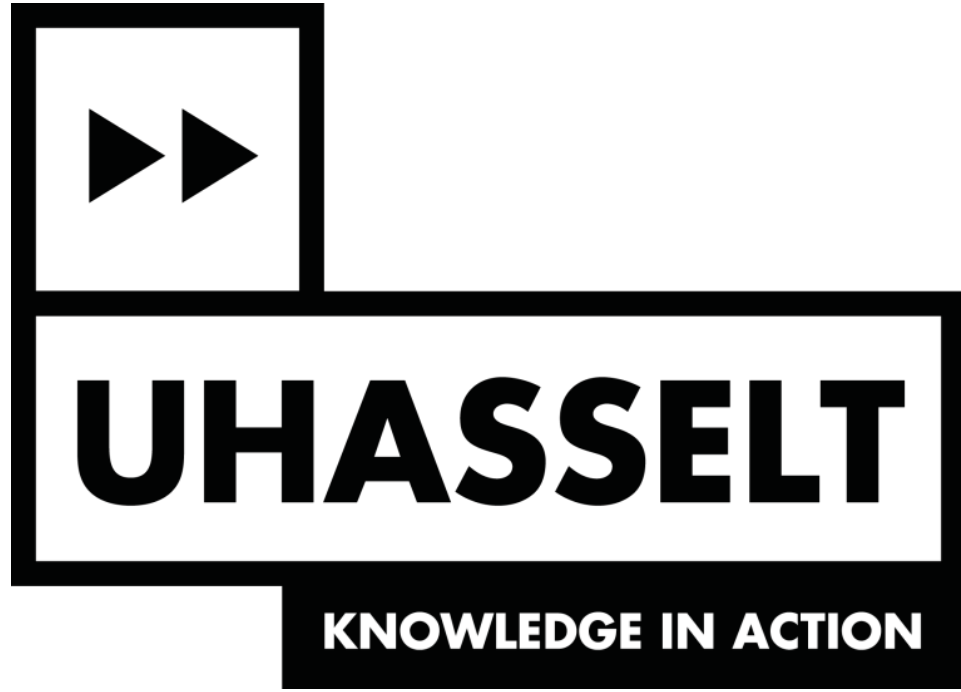

\#SPORTS KONGRES 2018

FEBRUARY $1-3$ COPENHAGEN

\section{Introduction}

The aims of this study were to examine the test-retest reliability of two-dimensional measured frontal and sagittal plane kinematics, and to determine how many steps should be included.

\section{Materials and Methods}

Twenty-one recreational runners (12 females, 9 males; mean $28.1 \pm 8.3$ years) participated in the study. All participants ran on a treadmill at their preferred speed (mean $10.2 \pm 1.2 \mathrm{~km} / \mathrm{h}$ ) and were tested twice (one-week interval). Digital videos were recorded in the frontal and sagittal plane with 2 iPads (Figure 1). The outcome measures were lateral trunk motion, contralateral pelvic drop, femoral adduction, hip adduction, foot and tibia inclination at initial contact, and knee flexion and ankle dorsiflexion during midstance (Figure 2). All angles were manually drawn using Kinovea during 10 consecutive steps for both legs. Intraclass correlation coefficients (ICC's), standard errors of measurement (SEM) and smallest detectable differences (SDD) were calculated (Table 1). A sequential estimation method was used to determine the number of steps needed to reach and maintain a stable mean.

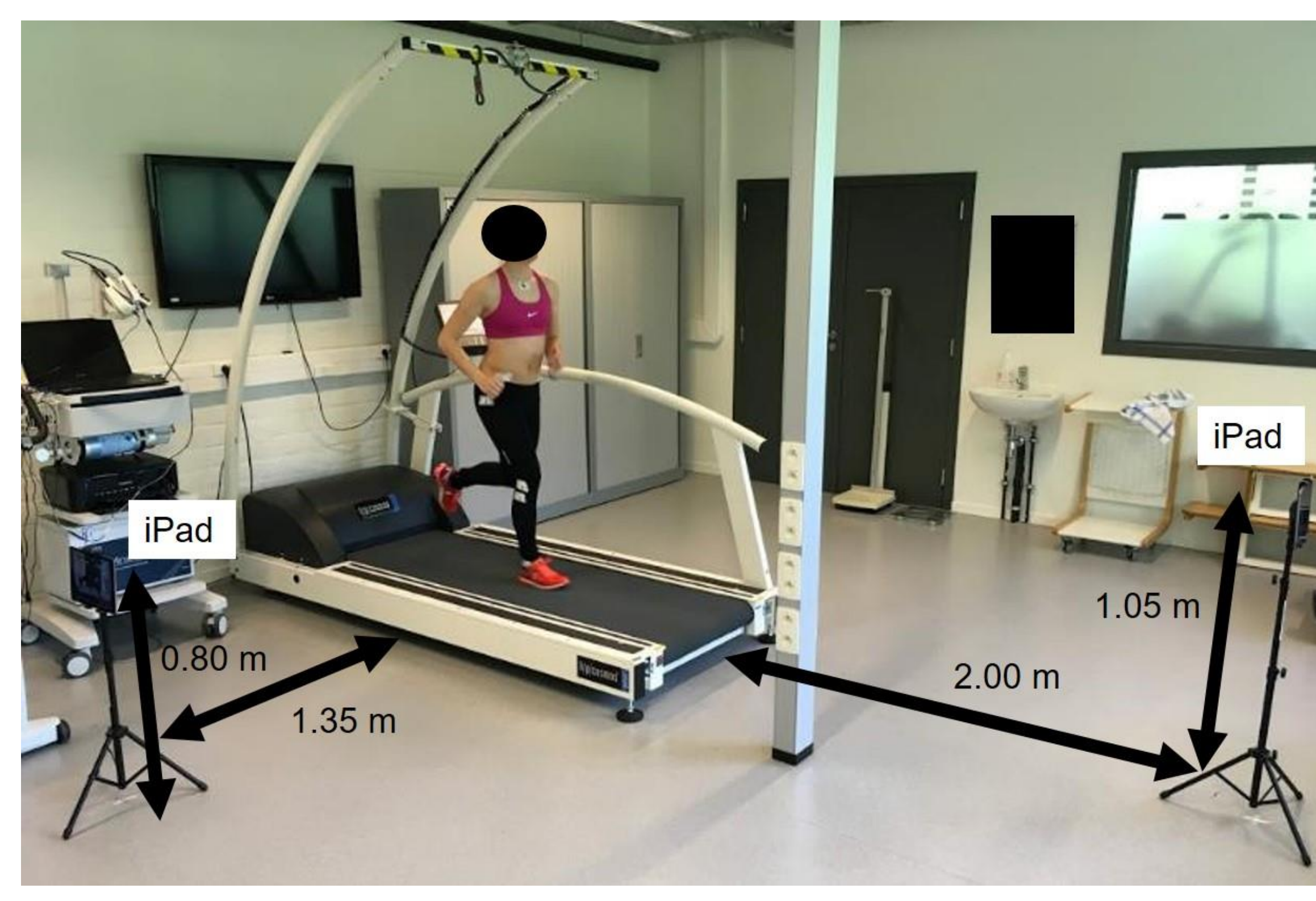

Figure 1. Experimental set-up.
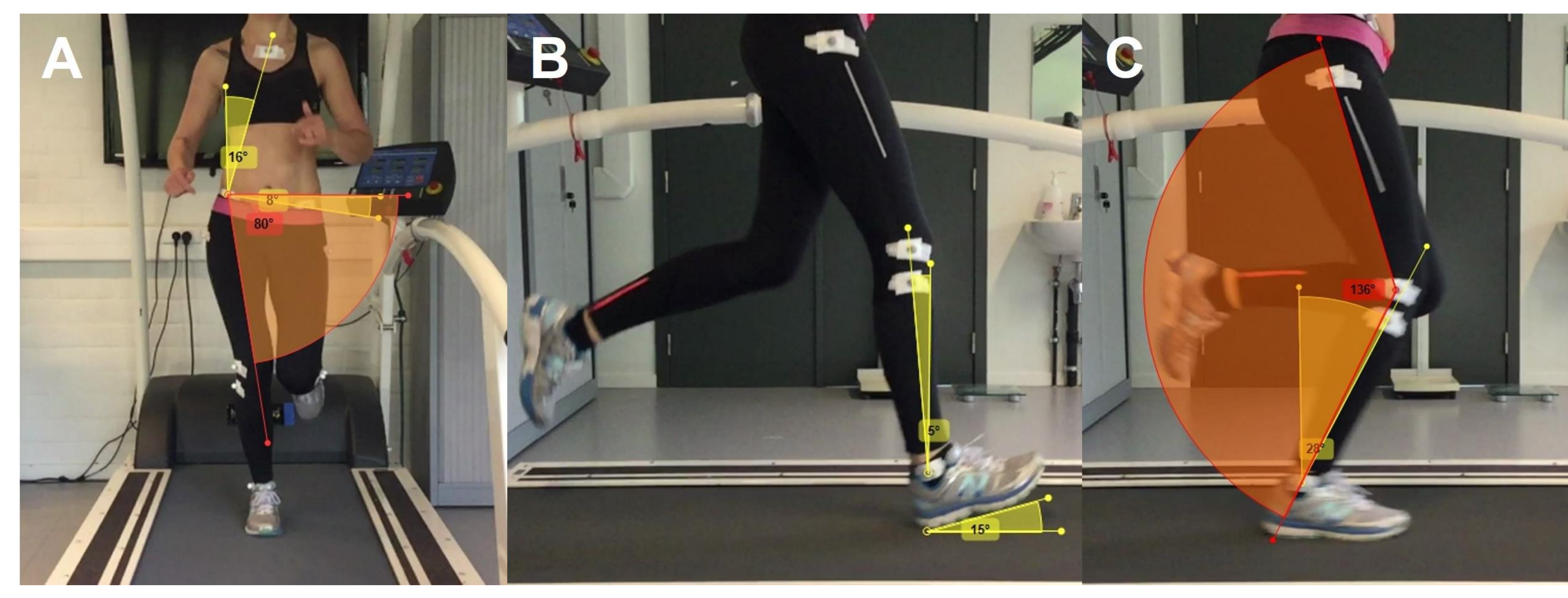

Figure 2. An example of the two-dimensional measurement of lateral trunk position, contralateral pelvic drop and femoral adduction (A), tibia inclination and foot inclination at initial contact $(B)$ and ankle dorsiflexion and knee flexion during midstance (C).

\section{Results}

The minimal number of steps needed to reach and maintain a stable mean ranged between 5.8 and 7.0. Across all angles of both legs, mean $\pm S D=6.3 \pm 0.3$ steps.

TABLE 1. Test-retest reliability of two-dimensional measured angles based on 6 steps

\begin{tabular}{|c|c|c|c|c|c|}
\hline Right leg & $\begin{array}{c}\text { Absolute difference } \\
\text { between measures }\left({ }^{\circ}\right)^{\star}\end{array}$ & $\mathrm{ICC}_{2,2}(95 \% \mathrm{Cl})$ & $\operatorname{SEM}\left({ }^{\circ}\right)$ & $\operatorname{SDD}\left({ }^{\circ}\right)$ & SDD/range (\%) \\
\hline Lateral trunk position & $1.0 \pm 0.7$ & $0.91(0.78-0.96)$ & 0.6 & 1.7 & 18.2 \\
\hline Contralateral pelvic drop & $1.2 \pm 1.1$ & $0.61(0.08-0.84)$ & 1.0 & 2.6 & 41.1 \\
\hline Femoral adduction & $0.9 \pm 0.6$ & $0.91(0.78-0.96)$ & 0.5 & 1.5 & 16.7 \\
\hline Hip adduction & $1.4 \pm 1.3$ & $0.83(0.60-0.93)$ & 1.0 & 2.8 & 27.5 \\
\hline Foot inclination & $1.2 \pm 1.0$ & $0.99(0.96-0.99)$ & 0.8 & 2.1 & 6.8 \\
\hline Tibia inclination & $1.3 \pm 0.8$ & $0.92(0.81-0.97)$ & 0.8 & 2.2 & 20.6 \\
\hline Knee flexion & $1.7 \pm 1.1$ & $0.87(0.68-0.95)$ & 1.0 & 2.9 & 19.8 \\
\hline Ankle dorsiflexion & $1.3 \pm 0.9$ & $0.90(0.74-0.96)$ & 0.8 & 2.3 & 24.6 \\
\hline Left leg & $\begin{array}{c}\text { Absolute difference } \\
\text { between measures }\left({ }^{\circ}\right)^{\star}\end{array}$ & $\mathrm{ICC}_{2,2}(95 \% \mathrm{Cl})$ & $\operatorname{SEM}\left({ }^{\circ}\right)$ & $\operatorname{SDD}\left({ }^{\circ}\right)$ & SDD/range (\%) \\
\hline Lateral trunk position & $1.0 \pm 0.7$ & $0.92(0.71-0.97)$ & 0.6 & 1.7 & 18.9 \\
\hline Contralateral pelvic drop & $1.4 \pm 1.0$ & $0.80(0.51-0.92)$ & 0.9 & 2.6 & 26.9 \\
\hline Femoral adduction & $0.8 \pm 0.5$ & $0.94(0.86-0.98)$ & 0.5 & 1.3 & 15.0 \\
\hline Hip adduction & $1.3 \pm 0.9$ & $0.87(0.69-0.95)$ & 0.8 & 2.3 & 21.5 \\
\hline Foot inclination & $1.4 \pm 0.9$ & $0.98(0.95-0.99)$ & 0.8 & 2.3 & 7.9 \\
\hline Tibia inclination & $2.1 \pm 1.2$ & $0.87(0.69-0.95)$ & 1.2 & 3.4 & 24.8 \\
\hline Knee flexion & $2.2 \pm 1.6$ & $0.89(0.71-0.95)$ & 1.4 & 3.8 & 22.8 \\
\hline Ankle dorsiflexion & $2.7 \pm 1.4$ & $0.75(0.37-0.90)$ & 1.7 & 4.7 & 34.0 \\
\hline
\end{tabular}

\section{Conclusion}

The results of the current study showed that:

- Two-dimensional video analysis can be reliably used to assess running kinematics, but the results differ between angles being measured.

- At least 6 steps should be included.

Corresponding author:

bart.dingenen@uhasselt.be 